\title{
Using off-the-Shelf Medical Devices for Biomedical Signal Monitoring in a Telemedicine System for Emergency Medical Services
}

\author{
Sebastian Thelen, Michael Czaplik, Philipp Meisen, Daniel Schilberg, and Sabina Jeschke, Senior Member, IEEE
}

\begin{abstract}
In order to study new methods of telemedicine usage in the context of emergency medical services, researchers need to prototype integrated telemedicine systems. To conduct a one-year trial phase-intended to study a new application of telemedicine in German emergency medical services-we used off-the-shelf medical devices and software to realize real-time patient monitoring within an integrated telemedicine system prototype. We demonstrate its feasibility by presenting the integrated real-time patient monitoring solution, by studying signal delay and transmission robustness regarding changing communication channel characteristics, and by evaluating issues reported by the physicians during the trial phase. Where standards like HL7 and the IEEE 11073 family are intended to enable interoperability of product grade medical devices, we show that research prototypes benefit from the use of web technologies and simple device interfaces, as they simplify product development for a manufacturer and ease integration efforts for research teams. Embracing this approach for the development of new medical devices eases the constraint to use off-the-shelf products for research trials investigating innovative use of telemedicine.
\end{abstract}

Index Terms-Biomedical telemetry, emergency medical services (EMS), telemedicine.

\section{INTRODUCTION}

$\mathbf{T}$ HE continued progress in mobile communication technology development is a driving force behind applications and research for telemedicine usage in emergency medical services (EMS). Many research projects have reported success in building telemedicine systems using different technologies to enable on-scene EMS staff to consult with specialists in a remote location. Some examples are [1]-[4]. In our pilot studies, the successful implementation of telemedicine systems for EMS, offering synchronous-often referred to as real-timetelemedical consultation functionality, has been demonstrated [5], [6].

Manuscript received March 27, 2014; revised July 31, 2014; accepted September 29, 2014. Date of publication October 8, 2014; date of current version December 30, 2014. This work was supported in part by EU/NRW Ziel 2-Program (EFRE) 2007-2013, the RWTH Aachen University, University Hospital Aachen, Philips HealthCare, 3M, and P3 communications.

S. Thelen, P. Meisen, D. Schilberg, and S. Jeschke are with the Institute of Information Management in Mechanical Engineering (IMA), RWTH Aachen University, 52068 Aachen, Germany (e-mail: sebastian.thelen@ima-zlw-ifu.rwthaachen.de; philipp.meisen@ima-zlw-ifu.rwth-aachen.de; daniel.schilberg@ ima-zlw-ifu.rwth-aachen.de; sabina.jeschke@ima-zlw-ifu.rwth-aachen.de).

M. Czaplik is with the University Hospital Aachen, 52074 Aachen, Germany, and also with the P3 telehealthcare $\mathrm{GmbH}$, Am Kraftversorgungsturm 3, 52070 Aachen, Germany and Docs in Clouds GmbH, Melanieweg 30, 52072, Aachen, Germany (e-mail: mczaplik@ukaachen.de).

Digital Object Identifier 10.1109/JBHI.2014.2361775
The telemedicine model applied in these two pilot studies has its origin in the German, physician-based, EMS system: two paramedics (an ambulance's crew in most German EMS) establish, either from a scene or from inside the ambulance, a telemedicine session with an especially trained EMS physician (hereafter called tele-EMS physician), who is located in a remote location (hereafter called teleconsultation center). During the telemedicine session, bidirectional real-time voice communication is the main information channel. Additionally, the tele-EMS physician receives information from a multitude of sources from the scene of incident and from within the ambulance: amongst others, the biomedical signals acquired by the patient monitoring device. In the teleconsultation center, two tele-EMS physicians are on call for involved ambulances; a tele-EMS physician supervises only a single ambulance team at once.

To collect evidence on the medical and organizational impact resulting from the use of this telemedicine model, the declared goal of our pilot studies was to conduct them in regular EMS operations. Consequently, the telemedicine system must not hinder patient treatment, making usability a major concern; besides, application of telemedicine must not prolong on-scene time intervals significantly, which was already analyzed in former studies [7]. In addition to usability, organizational aspects and statutory regulations of medical devices are of major importance to the technical system realization [8]-[10]. Philips Healthcare (Boeblingen, Germany) joined the research project and supported it by providing off-the-shelf medical devices for patient monitoring (Philips MRx monitoring/defibrillator device) and accompanying software. From a technical perspective, the challenge to incorporate these devices into an integrated telemedicine system-together with additional communication technology and custom software-remained.

We have already reported general results from a one-month test phase of this integrated telemedicine system in [11], where we discuss overall requirements and the performance we achieve with the overall system architecture. Still, we have not yet reported on the solution to the aforementioned integration challenge, which is a major enabler of our study's trail phase and as such may provide valuable insight for future telemedicine projects in EMS.

During our work, we realized that the most challenging system integration aspect is the biomedical real-time signal transmission for continuous patient monitoring and diagnostics. Current literature leaves central questions unanswered regarding this topic: How can this integration be achieved, what is the 
impact of existing interoperability standards, and what performance regarding usability, robustness, and real-time aspects are achievable? To address these open issues, this paper is organized as follows. In Section II, we summarize related work. In Section III, we present the requirements that the integrated biomedical signal monitoring has to fulfill. In Section IV, we present our system design that integrates the biomedical signal monitoring. In Section V, we present the performance evaluation. In Section VI, we discuss the results of our performance evaluation and the impact of relevant interoperability standards. In Section VII, we draw the conclusion of our work presented in this paper.

\section{RELATED WORK}

Research in mobile telemedicine systems has been broadly surveyed by others; for an overview, we refer to [12] and instead focus on the works most relevant for this paper, i.e., those that contribute important details to biomedical signal transmission for patient monitoring in EMS telemedicine systems. Lin [12] offered a broad technology-focused survey of telemedicine research; he discusses application areas, communication technology, and the kind of data that are transmitted. From the projects he describes, two are of concrete relevance for our work: Anogianakis et al. [13] described the early maritime telemedicine system MERMAID that enables teleconsultation centers to provide medical assistance for the patient treatment on board of ships using satellite communication. Kyriacou et al. [14] reported on a real-time telemedicine system for use in EMS, the general concept being close to our use of telemedicine; however, the integration of biomedical signal transmission differs. They access monitoring data directly at the patient monitoring devices using a RS-232 interface and custom transmission algorithms and viewing applications. Thereby they effectively implement software that has to be classified as active medical device, if intended for patient diagnostics [10].

Alesanco and Garcia [15] analyzed the transmission of clinical real-time electrocardiogram (ECG) via $3 \mathrm{G}$ mobile networks using simulations. They configure their proposed transmission algorithm - real-time/reliable ECG transmission protocol-in such a way, that it maintains a signal delay below 3-4 s. According to their clinical trials, $4 \mathrm{~s}$ is the maximum acceptable delay. Trigo et al. [16] presented a prototype implementation of real-time ECG transmission using the IEEE 11073 standards family, while harmonization for the ECG device specialization was still ongoing. This prototype is not clinically evaluated, but demonstrates general feasibility. The IEEE 11073 ECG device specialization standard was published in 2012 [17].

LifeBot DREAMS is a commercially advertised, mobile EMS telemedicine system in the US [18], [19]; technical details and findings for use by other telemedicine studies are not reported. Similarly, Viewcare is a Danish telemedicine initiative that utilizes telemedicine in ambulance vehicles [20]; detailed information is scarce as well.

\section{SYSTEM REQUIREMENTS}

In a multidisciplinary team of emergency physiciansexperienced in telemedicine usage from a former study-, engineers, and organizational experts, we conducted a thorough requirements analysis before starting to design the system. To document the requirements in a structured and comprehensive way, we used use-case descriptions. An iterative design process ensured that the requirements, user expectations, and system development remained aligned. In [21], we have given a general overview of the system requirements, but because of space limitations, we neglected specific details necessary for the discussion in this paper; the functional requirements that the biomedical signal monitoring integration has to fulfill are as follows:

1) minimal need for an additional system interaction onscene, after a teleconsultation has been successfully initiated, e.g., data transmission either happens automatically or is controlled by the tele-EMS physician, not by the on-scene paramedics;

2) to protect patient and staff privacy, no data are transmitted without an active teleconsultation session;

3) to minimize potential for confusion during a teleconsultation session, only data from the active case may be displayed to the tele-EMS physician;

4) the tele-EMS physician must be able to switch the currently active, i.e., displayed, case by selecting the target ambulance with a single user interface interaction, e.g., a single button press and potentially a confirmation;

5) continuous transmission of at least two ECG leads and pulse oximetry $\left(\mathrm{SpO}_{2}\right)$ waveforms as well as numeric values of heart rate, $\mathrm{SpO}_{2}$, pulse, and blood pressure with minimal achievable delay, ideally not above $3 \mathrm{~s}$;

6) transmission of 12-lead ECG recordings;

7) periodic transmission of numeric trend values for heartrate, $\mathrm{SpO}_{2}$, and pulse with a 1-min interval, in case the network conditions on-scene are too bad to support requirement 5;

8) all data acquired during a teleconsultation session, such as the biomedical signals from requirements 5-7, must be available in the application that the tele-EMS physicians uses to create the documentation of the teleconsultation session.

\section{SySTEM DESIGN OF INTEGRATED BIOMEDICAL SIGNAL MONITORING}

In [11] and [21], we already introduced the general system architecture, but did not discuss the design of the biomedical signal transmission for patient monitoring, its real-time performance, nor our findings in regard to its applicability for future telemedicine projects. The-commonly used-combined patient monitor and defibrillator HeartStart MRx (model M3535A, Philips Healthcare, Andover, MA, USA) is the device we chose for integration into the system; the biomedical signals that this device acquires and that are relevant to our work are listed in Table I, together with the communication interfaces used to access them. In addition to the HeartStart MRx, we tested our integration approach with the multiparameter monitor IntelliVue X2 (Philips Healthcare, Boeblingen, Germany). The HeartStart MRx can be equipped with different configuration options that provide different interfaces and communication 
TABLE I

RELEVANT BIOMEDICAL Signal TRANSMISSION CAPABILITIES OFFERED BY HEARTSTART MRX

\begin{tabular}{|c|c|c|}
\hline Signal & TRANSMISSION PERIODICITY & Communication Interface \\
\hline $\begin{array}{l}\text { waveforms: two-lead } \\
\text { ECG, pulse oximetry } \\
\left(\mathrm{SpO}_{2}\right)\end{array}$ & continuous, real-time & $\begin{array}{c}\text { Internet Protocol (IP) via } \\
\text { Ethernet }\end{array}$ \\
\hline $\begin{array}{l}\text { numeric values: } \\
\text { heart-rate, } \mathrm{SpO}_{2} \text {, pulse, } \\
\text { blood-pressure }\end{array}$ & on-change & IP via Ethernet \\
\hline $\begin{array}{l}\text { numeric values: } \\
\text { heart-rate, } \mathrm{SpO}_{2} \text {, pulse, } \\
\text { blood-pressure }\end{array}$ & periodically: once per minute & $\begin{array}{l}\text { Bluetooth File Transfer } \\
\text { Profile }\end{array}$ \\
\hline $\begin{array}{l}\text { 12-lead ECG }(3.3 \mathrm{~s} \\
\text { recording) }\end{array}$ & on-capturing/on-demand & $\begin{array}{c}\text { Bluetooth File Transfer } \\
\text { Profile }\end{array}$ \\
\hline
\end{tabular}

capabilities. Relevant for our system integration are the options IntelliVue Net (continuous real-time waveform transmission in a local area network (LAN)), 12-LTx Bluetooth (transmission of 12-lead ECG recordings via Bluetooth), and Per Data Tx (periodic transmission of biomedical signal's numeric values via Bluetooth).

The IntelliVue Net option, which provides access to a patient's biomedical real-time vital signals, usually utilizing a direct LAN connection to the proprietary, combined software/hardware appliance IntelliVue Information Center (hereafter called IIC; Philips Healthcare, Andover, MA, USA) for signal transmission [22], whereas the other two options wirelessly push data via Bluetooth to a connected device. The latter data can be automatically imported into and displayed by the proprietary software HeartStart Telemedicine System (hereafter called HTS; Philips Healthcare, Andover, MA, USA); alternatively, the HeartStart DataSDK (Philips Healthcare, Andover, MA, USA) - a software development kit-may be used to implement import adapters that enable access to this data from custom applications [23].

Both the IIC and the HTS are certified medicine products and their intended use covers patient diagnostics [22], [23]. In the integrated telemedicine system, the use of periodic data transmission is-according to requirement 7-solely considered as a fallback option. While no single integration option fulfills the aforementioned requirements, in combination they cover the requirements 5 to 7: IntelliVue Net option for requirement 5, 12-LTX Bluetooth option for requirement 6, and Per Data-Tx for requirement 7 . Because of their different underlying interface technologies and differing proprietary display applications, we had to implement two distinct integration strategies as shown in Fig. 1. For the continuous real-time waveforms and numeric values of requirement 5 , we used networking technologies, and for the 12-lead ECG and periodic data transmission of requirement 6 and 7, we used Bluetooth and file transfer services. In order to fulfill the remaining requirements 1 to 5 , we had to integrate both IIC and HTS into the tele-EMS physician's workplace and automate the process of switching the currently displayed monitoring devices as well as the activation and deactivation of data transmission from the scene. Fig. 2 shows the various components and their connections that implement the integration strategies. In the remainder of this section, we provide further detail to the system integration.

\section{A. Mobile Communication Unit}

The Mobile Communication Unit (B in Fig. 2) securely connects all on-scene devices to the teleconsultation center; it simultaneously uses three dedicated mobile network data connections (C) to create a single, encrypted Internet protocol (IP) connection to the stationary communication unit (D) via the Internet. This connection forms a secured virtual private network (VPN), containing the on-scene and the teleconsultation center IP networks.

The mobile communication unit's three mobile network modems support both 2G (GPRS and EDGE) and 3G (UMTS, HSDPA, and HSUPA) data connections, individually choosing the fastest network technology that is available [24]. In order to ensure on-scene usability, the mobile communication unit is housed inside the patient monitor's bag, as shown in Fig. 1, that was modified to compensate for the space required by the mobile communication unit.

\section{B. Continuous, Near Real-Time Signal Transmission}

The transmission of continuous, near real-time waveform signals is realized by connecting the patient monitor (A) via Ethernet to the mobile communication unit. The mobile communication unit connects the patient monitor to the clinical network of the IIC (F) using an additional VPN from Mobile Communication Unit to Stationary Communication Unit to create an Ethernet bridge on the data-link layer. The data-link layer firewall (E) generally blocks communication between connected patient monitors and the IIC. In this default setting, the patient monitor is unable to establish a connection to the IIC (requirement 2). To enable the connection and transmission of the biomedical signals, the tele-EMS physician selectively activates each patient monitor's connection (requirement 1). This setup prevents unintended data transmission and resulting privacy concerns, because the monitoring device would otherwise begin to transmit signals to the IIC as soon as it is turned ON, even without an active teleconsultation session.

On the tele-EMS physician workplace $(\mathrm{K})$, the data transmitted from the on-scene patient monitor is accessible through the IIC's intranet patient viewer web-application. This web-application's monitoring widget—displaying a patient's biomedical signal waveforms and numeric values - is embedded into a wrapper web application. The wrapper web application selects the patient to be viewed when loading the embedded monitoring widget without additional interaction by the teleEMS physician (requirements 3 and 4). Direct access to the numerical values and trend data for the waveform signals is enabled via the IIC's HL7 unsolicited messages interface that publishes data in a configurable interval. These data are then forwarded by a custom import adapter into the case documentation (requirement 8).

\section{Periodic Signal Transmission}

For transmission of 12-lead ECG recordings and periodic trend values of a patient's biomedical signals, the patient monitor (A) connects to the mobile communication unit (B) via Bluetooth using the Bluetooth File Transfer Profile. For each 


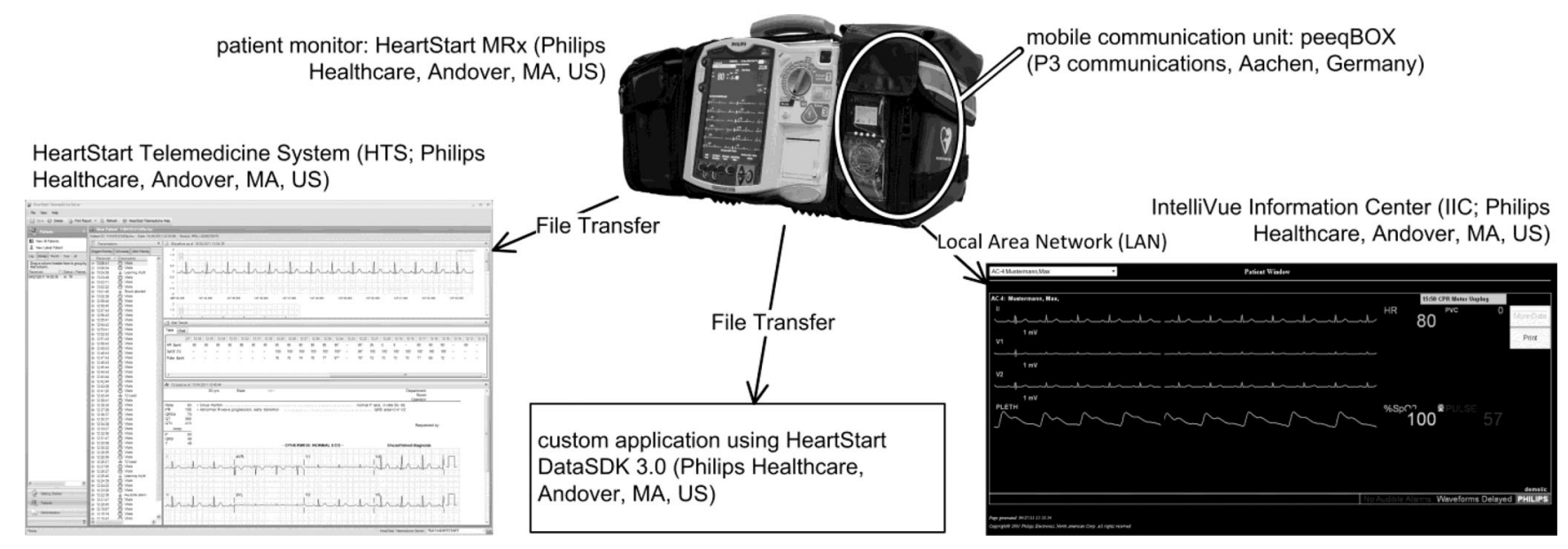

Fig. 1. Device and application overview for the integrated biomedical signal monitoring system.

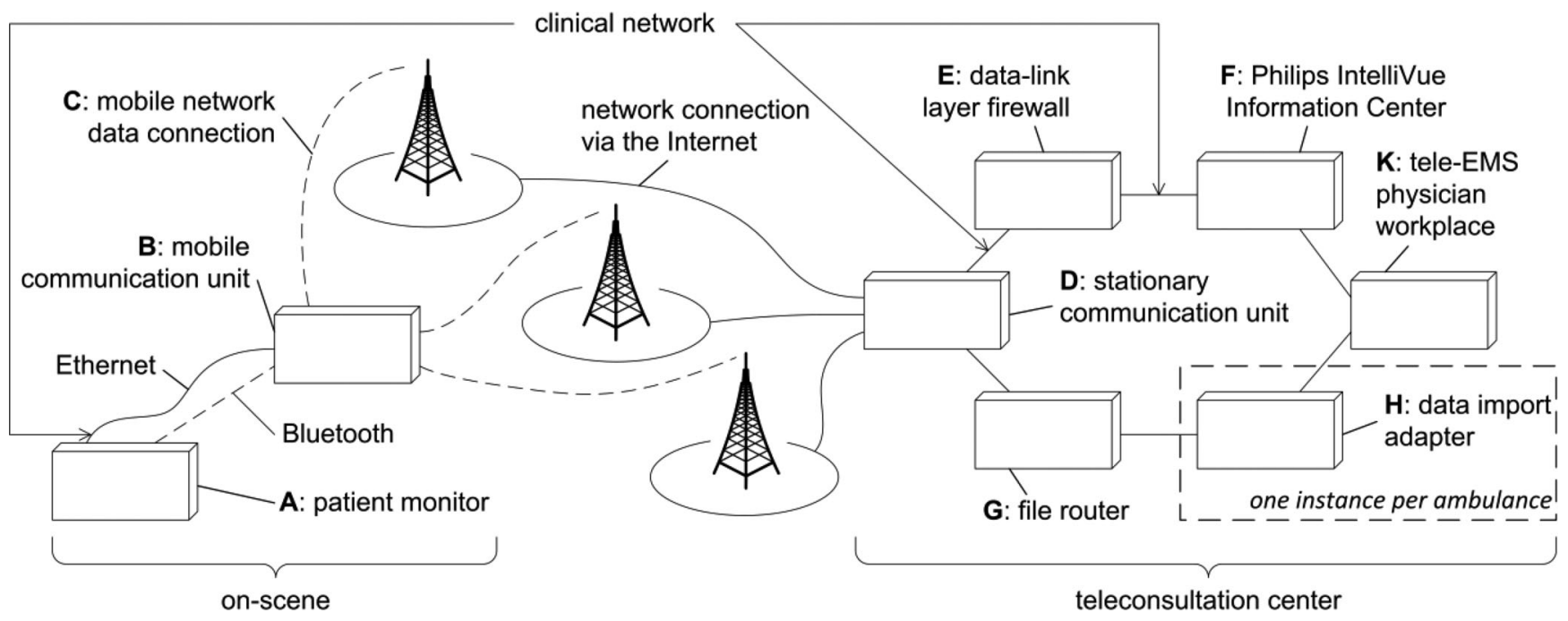

Fig. 2. Components and communication links in the integrated biomedical signal monitoring system.

data transmission, i.e., a single 12-lead ECG record or a set of biomedical signal trend values, the patient monitor transmits three files to the mobile communication unit via this Bluetooth connection.

The Mobile Communication Unit forwards these received files using File Transfer Protocol (FTP) to the Stationary Communication Unit (D), which in turn forwards these files again via FTP to the file router (G). Here, these files are delivered, again via FTP, to two distinct data directories on the data import adapter instance $(\mathrm{H})$ that is assigned to the originating ambulance. Both data directories of a data import adapter instance are watched by a separate data import service. One data import service is the HTS, which imports all incoming data into its case database, the other is a custom data import application implemented using the DataSDK, which forwards the data to the case documentation (requirement 8). The way that the HTS displays transmissions-grouped to cases from all connected patient monitors in a single list—contradicts requirement 3. Our solution is to use a separate HTS instance for each ambulance in the system, and thus, one data import adapter $(\mathrm{H})$ per ambulance, in order to fulfill requirement 3 .

On the tele-EMS physician workplace (K), the HeartStart Telemedicine Viewer (hereafter called HTS Viewer; Philips Healthcare, Andover, MA, USA) - a proprietary, dedicated application that connects to one HTS at a time and allows remote access to the HTS with an identical user interface-is connected to the HTS instance associated to the current ambulance. By utilizing a dedicated HTS instance per ambulance, the list of transmissions that the HTS viewer presents to the tele-EMS physician contains only one active case. We were unable to automate the selection of this active case, thus the tele-EMS physician must manually select the sole active case, partially violating requirement 3 . In order to display the transmissions from another ambulance, the HTS viewer must be connected to the HTS associated with the desired ambulance, a task we were able to automate using the user interface automation tool AutoIt 
TABLE II

THRESHOLD VALUES OF COMMUNICATION CHANNEL CHARACTERISTICS FOR STABLE ECG AND $\mathrm{SPO}_{2}$ WAVEFORM TRANSMISSIONS

\begin{tabular}{lc}
\hline \hline Channel Parameter & Threshold Value \\
\hline delay & $3.15 \mathrm{~s}$ \\
jitter (with 1 s base delay) & $0.5 \mathrm{~s}$ \\
data loss & $27 \%$ \\
data corruption & $24 \%$ \\
\hline \hline
\end{tabular}

(AutoIt Consulting, GB), fulfilling requirement 4 . To our knowledge, there is no programming interface available to control the HTS Viewer.

\section{Evaluation Methods And Results}

In this paper, we focus on two major aspects of our evaluation of the presented biomedical signal monitoring system: the system's real-time performance in Section V-A and the usability and robustness we achieve with our system integration in Section V-B. Outside of the scope of this paper is to report more broadly about the one-year trail phase that we used to evaluate the complete telemedicine system and its organizational and medical procedures, which ended in July 2013: six ambulance teams in five different EMS departments were using the system during their regular EMS operations; two tele-EMS physicians staffed the teleconsultation center during this time. A one-month field test before starting the trail phase ensured that the system technically performed as necessary. The physicians carrying out the field test evaluation (age 33 to 44, assigned as EMS physician for 3 to 14 years) rated the system performance as satisfactory, thus the trial phase could start, as we reported in [11].

\section{A. Continuous, Near Real-Time Signal Monitoring}

Responsible for the near real-time biomedical signal monitoring is the IIC and its connection to the patient monitor. To establish baseline parameters about this connection's robustness against failures in the underlying communication channel, i.e., delay, jitter, data loss, and data corruption, we connected the patient monitor via Ethernet to the IIC's clinical network and injected a network emulator, [25], into this Ethernet connection. The three parameters were then individually increased, starting at zero, with the other parameters held at zero; the threshold value, above that at least one of the ECG or the $\mathrm{SpO}_{2}$ waveforms started showing the slightest form of interruptions, i.e., missing data points, is recorded (see Table II).

The end-to-end, i.e., patient monitor to tele-EMS physician display, signal delay for transmission of continuous, near realtime ECG and $\mathrm{SpO}_{2}$ waveforms was measured by a test engineer, who directly observed the waveforms displayed on the patient monitor as well as on the tele-EMS physician's workplace display; while transmitting regular signals, either from a patientsimulation device (ECG) or a test subject's finger $\left(\mathrm{SpO}_{2}\right)$, a clearly perceivable change in the signal was triggered; the time difference was measured from onset of this change on the patient monitor's display to its occurrence on the tele-EMS physician's

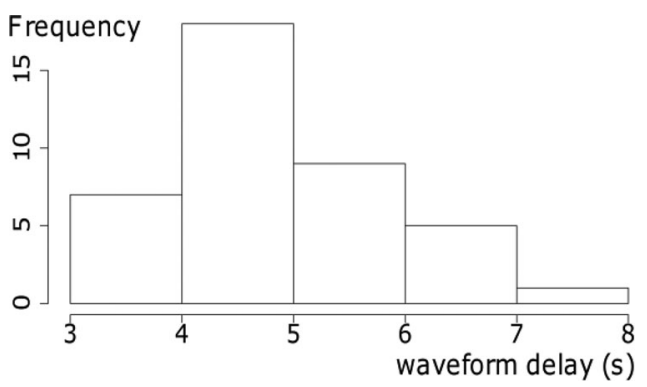

Fig. 3. Grouped histogram of ECG and $\mathrm{SpO}_{2}$ signal's waveform end-to-end delay between occurrence on the patient monitor display and the graphical user interface of the teleconsultation center.

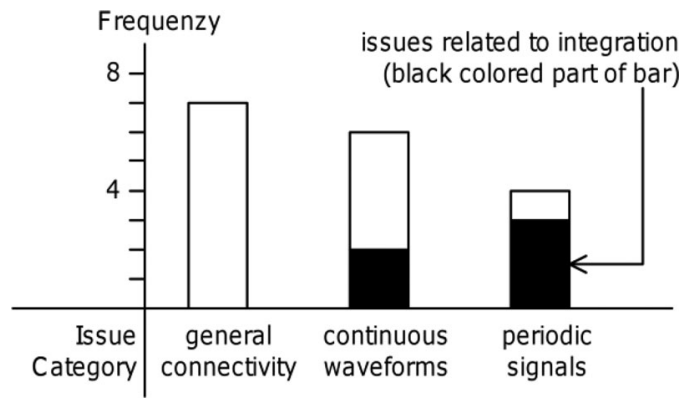

Fig. 4. Histogram of defect issues concerning the biomedical signal monitoring as reported during the one-year trial phase, grouped by issue category.

workplace display. For each signal type (ECG and $\left.\mathrm{SpO}_{2}\right)$, the delay was independently measured ten times when the mobile communication unit simultaneously used three $3 \mathrm{G}$ data connections (signal strengths at $77-87 \%$ / 61-74\% / 35-45\%) and ten times, when it simultaneously used three $2 \mathrm{G}$ data connections (signal strengths at $93-96 \% / 70-77 \%$ / 58\%).

The grouped histogram of all 40 samples is shown in Fig. 3. The signal in the teleconsultation center is delayed with a median of $4.9 \mathrm{~s}$ (range 3.4-7.1 s); no correlation with the signal type $\left(\mathrm{ECG}\right.$ and $\mathrm{SpO}_{2}$ ) or the data connection's technology ( $2 \mathrm{G}$ and $3 \mathrm{G})$ exists. Due to this range of measurements and with an additional security factor applied, tele-EMS physicians were instructed to expect a maximum signal delay of $10 \mathrm{~s}$, when instructing patient treatment.

\section{B. Usability and System Integration}

During the one-year trial phase, the tele-EMS physicians and the technical support team gathered quantitative data regarding technical system performance in a ticket system to track the occurrence and elimination of technical performance issues. Out of the 377 issues reported as defects, 17 were related to the biomedical signal monitoring. These 17 issues (see Fig. 4) can be categorized into three groups: general connectivity (seven issues), continuous waveforms (six issues), and periodic signals (four issues). The general connectivity issues describe unacceptable amount or length of dropouts for continuous waveforms and unacceptable delay of transmitted 12-lead ECGs, all 
caused by poor mobile data connectivity from on-scene to the teleconsultation center.

The continuous waveform issues were largely caused by unexpected behavior of the embedded IIC display component ( 4 out of 6 issues, i.e., $66.7 \%$ ), for which no causes could be identified: the color-coded waveforms and numerical values were suddenly all displayed white, but could still be read (independently reported twice); the component's display froze and it became unusable; no waveforms, only the numerical values were displayed. The remaining issues were caused by errors in the custom integration software, failing to handle unexpected situations (two issues).

Most issues ( 3 out of 4 issues, i.e., $75 \%$ ) related to the periodic signals were caused by the custom integration software failing to control the HTS Viewer on a tele-EMS physician's workplace to connect to the correct HTS; the remaining issue was caused by the HTS Viewer staying in an unusable state for about one minute when connecting to an HTS with many stored cases.

\section{DISCUSSION}

Using off-the-shelf medical devices and software to build an integrated telemedicine system proved successful. The presented integration approach fulfills the functional requirements that a multidisciplinary team of experts from within the domain of telemedicine in EMS specified prior. The completion of a oneyear trial phase in regular EMS, using this system, validates both the technical system and the requirements upon which it is built. This trial enabled medical researchers to gather data to assess the effectiveness of the underlying model for telemedicine and its organizational impact on EMS. The medical trial evaluation is not yet finished but initial analysis is promising [6], [11].

Comparing the doubled maximum channel delay tolerated by the patient monitor to IIC connection $(6.3 \mathrm{~s})$ to the maximum round trip time expected in GPRS (mean below $2 \mathrm{~s}, 95 \%$ percentile can increase up to $4 \mathrm{~s}$ ) and UMTS (mean at about $0.5 \mathrm{~s}$, 95\%-percentile at about $1 \mathrm{~s}$ ) networks [26], you find evidence that a GPRS network is sufficient for near real-time biomedical signal transmission by using the presented setup in most circumstances, given an adequate signal strength and link quality. In the fully integrated telemedicine system, the negative effects of data loss and data corruption are prevented by the VPN tunnel using a reliable transport protocol; this in turn, adds to the channel's delay and causes more jitter. Considering our findings from [11], some rural areas exist that do not provide sufficient network connectivity to support adequate real-time monitoring using this setup and that require the backup mechanism provided by the HTS integration.

The measured end-to-end delay for near real-time biomedical signal waveforms exceeded the otherwise suggested maximum delay of $4 \mathrm{~s}$ in $82.5 \%$ of the samples, with the median exceeding this suggested delay by $0.9 \mathrm{~s}$ [15]. Regarding the $10 \mathrm{~s}$ expected delay that was used to instruct the tele-EMS physicians for the trial phase, it is unclear, if a generalized requirement can be upheld. However, in diverse clinical circumstances a delay of $10 \mathrm{~s}$ seems to be rather inacceptable, e.g., when ECG suddenly presents an asystole that actually occurred already $10 \mathrm{~s}$ ago.
Furthermore, communication between the tele-EMS physician and the paramedics concerning alterations in real-time ECG curve (e.g., extra systoles) can be hindered.

The technical integration issues reported during the trail phase fall into two categories: the issues that can be resolved by improving the custom integration software and the issues that result from insufficient means of integration offered by the proprietary application. The two integration issues reported for the IIC fall into the former category and can be eliminated in a future system, the three integration issues reported for the HTS fall into the latter category. The IIC's web technology based interface allows a more stable integration, because of its individually addressable and embeddable user interface components. Contrary, the attempt to integrate the native, stand-alone HTS viewer application using user interface automation proved to be an unreliable method.

All reported issues for the patient monitoring but the two IIC issues with missing color, severely impact the diagnostic capabilities that the telemedicine system offers to the tele-EMS physician. The issue of HTS staying in an unusable state could be solved by backing up old cases and removing them from the main database. Nevertheless, the tele-EMS physician's most important source of information is the bidirectional voice communication to the paramedics on-scene, which was reported as at least adequate even under bad conditions [11]. Hence, a defect in the patient monitoring is not a life threatening situation for a patient in this telemedicine setting.

\section{CONCLUSION}

We described how to use a commercially available off-theshelf monitor/defibrillator (HearStart MRx, Philips Healthcare, Andover, MA, USA) and related software to create an integrated telemedicine system prototype, sufficiently fulfilling requirements regarding statutory regulations, usability, and functionality, in order to enable research of telemedicine in regular EMS. Despite numerous existing medical data and interface standards, only HL7 messaging was of importance for the integration; otherwise, web and common computer networking technology offered good integration methods. While our work here focuses on integrating one device, our general approach of integrating off-the-shelf components instead of building a complete system from scratch can speed up the creation of prototypes for research in clinical trials.

Standards such as HL7 and IEEE 11073 promise full interoperability between medical devices and external software systems; with our work, we have shown that, in order to easily create an integrated telemedicine system prototype for clinical research, a full implementation of these standards is not necessary.

Building user interfaces with standard web technologies that offer individually addressable display components vastly eases the work of researchers, who aim to evaluate new methods for utilizing existing technology. On the data interface level, using common computer standards and application protocols-in our case Bluetooth File Transfer Profile together with messages stored in files-offers an integration point for nonreal-time data 
that is very simple to access. A device manufacturer searching for a simple device interface, unwilling or unable to use IEEE 11073, should consider Bluetooth File Transfer Profile instead of using a proprietary protocol implemented on top of other Bluetooth Profiles.

At this point, very little work has been done to systematically identify which biomedical signals (and signal qualities) actually are necessary for specific telemedicine procedures in EMS. The aspect of signal delay in this regard has been briefly touched by our work, but clearly, more research directed to this special aspect is necessary. Resulting knowledge would drastically improve the support a telemedicine system can offer to a teleEMS physician who has to decide for a treatment method that heavily relies on the availability of biomedical signals within a certain maximum delay; this very specific maximum delay then defines real-time for the case at hand, which can automatically be assessed by the telemedicine system.

\section{REFERENCES}

[1] S. Pavlopoulos, E. Kyriacou, A. Berler, S. Dembeyiotis, and D. Koutsouris, "A novel emergency telemedicine system based on wireless communication technology-AMBULANCE," IEEE Trans. Inf. Technol. Biomed, vol. 2, no. 4, pp. 261-267, Dec. 1998.

[2] F. Chiarugi, D. Trypakis, V. Kontogiannis, P. Lees, C. Chronaki, M. Zeaki, N. Giannakoudakis, D. Vourvahakis, M. Tsiknakis, and S. Orphanoudakis, "Continuous ECG monitoring in the management of pre-hospital health emergencies," in Computers in Cardiology, Piscataway, NJ, USA: IEEE, 2003, pp. 205-208.

[3] C.-S. Chang, T.-H. Tan, Y.-F. Chen, Y.-F. Huang, M.-H. Lee, J.-C. Hsu, and H.-C. Chen, "Development of a ubiquitous emergency medical service system based on zigbee and 3.5g wireless communication technologies," in Medical Biometrics (Lecture Notes in Computer Science), vol. 6165, D. Zhang and M. Sonka, Eds. Berlin, Germany: Springer, 2010, pp. 201208.

[4] K. Shimizu, "Telemedicine by mobile communication," IEEE Eng. Med. Biol. Mag., vol. 18, no. 4, pp. 32-44, Jul./Aug. 1999.

[5] S. Bergrath, D. Rörtgen, R. Rossaint, S. K. Beckers, H. Fischermann, J. C. Brokmann, M. Czaplik, M. Felzen, M.-T. Schneiders, and M. Skorning, "Technical and organisational feasibility of a multifunctional telemedicine system in an emergency medical service-An observational study," J. Telemed. Telecare, vol. 17, no. 7, pp. 371-377, Oct. 2011.

[6] S. Bergrath, M. Czaplik, R. Rossaint, F. Hirsch, S. Beckers, B. Valentin, D. Wielpütz, M.-T. Schneiders, and J. Brokmann, "Implementation phase of a multicentre prehospital telemedicine system to support paramedics: Feasibility and possible limitations," Scand J. Trauma. Resusc. Emerg. Med., vol. 21, no. 54, pp. 1-10, 2013.

[7] M. Skorning, S. Bergrath, D. Rörtgen, S. K. Beckers, J. C. Brokmann, B. Gillmann, J. Herding, M. Protogerakis, C. Mutscher, and R. Rossaint, "Teleconsultation does not prolong time intervals in a simulated prehospital cardiac emergency scenario," Circulation, vol. 120, no. S1482, 2009.

[8] M.-T. Schneiders, D. Schilberg, and S. Jeschke, "A joint organizational and technical development of a telematic rescue assistance system for German emergency medical services," in Proc. 3rd Int. Conf. eHealth, Telemed., Social Med., Gosier, Guadeloupe, France, 2011, pp. 150-155.

[9] F. Hulstaert, M. Neyt, I. Vinck, S. Stordeur, M. Huic, S. Sauerland, M. R. Kuijpers, P. Abrishami, H. Vondeling, and $\mathrm{H}$. van Brabandt, "The pre-market clinical evaluation of innovative high-risk medical devices," Health Service Research (HSR), Belgian Health Care Knowledge Centre (KCE), Brussels, Belgium KCE Report 158C, 2011.
[10] E. French-Mowat and J. Burnett, "How are medical devices regulated in the European Union?” J. Roy. Soc. Med., vol. 105, pp. S22-S28, 2012.

[11] M. Czaplik, S. Bergrath, R. Rossaint, S. Thelen, T. Brodziak, B. Valentin, F. Hirsch, S. K. Beckers, and J. C. Brokmann, "Employment of telemedicine in emergency medicine," Methods Inf. Med., vol. 53, no. 2, 2014.

[12] C.-F. Lin, "Mobile telemedicine: A survey study," J. Med. Syst., vol. 36, no. 2, pp. 511-520, Apr. 2012

[13] G. Anogianakis, S. Maglavera, A. Pomportsis, S. Bountzioukas, F. Beltrame, and G. Orsi, "Medical emergency aid through telematics: Design, implementation guidelines and analysis of user requirements for the MER MAID project," Int. J. Med. Inform., vol. 52, no. 1-3, pp. 93-103, Oct. 1998.

[14] E. Kyriacou, S. Pavlopoulos, A. Berler, M. Neophytou, A. Bourka, A. Georgoulas, A. Anagnostaki, D. Karayiannis, C. Schizas, C. Pattichis, A. Andreou, and D. Koutsouris, "Multi-purpose healthcare telemedicine systems with mobile communication link support," BioMed. Eng. OnLine, vol. 2 , no. 7, 2003.

[15] A. Alesanco and J. García, "Clinical assessment of wireless ECG transmission in real-time cardiac telemonitoring," IEEE Trans. Inf. Technol. Biomed., vol. 14, no. 5, pp. 1144-1152, Sep. 2010.

[16] J. D. Trigo, F. Chiarugi, A. Alesanco, M. Martínez-Espronceda, C. E. Chronaki, J. Escayola, I. Martínez, and J. García, "Standardcompliant real-time transmission of ECGs: Harmonization of ISO/IEEE 11073-PHD and SCP-ECG," in Proc. IEEE Annu. Int. Conf. Eng. Med. Biol. Soc., 2009, pp. 4635-4638.

[17] Health informatics-Personal health device communication-Part 10406 Device specialization - Basic electrocardiograph (ECG) (1- to 3-lead ECG), IEEE Standard 11073-10406, 2012.

[18] LifeBot. (2012, Sep. 27). LifeBot DREAMS: The Most Advanced Telemedicine Systems. [Online]. Available: http://www.lifebot. us.com/dreams/

[19] Resuscitation and life support system, method and apparatus, by R. L. Heath, Patent US 7672 720, (2010, Mar. 2).

[20] Viewcare. (2013, Oct. 23). Viewcare Pre-Hospital Telemedicine System. [Online]. Available: http://viewcare.com/index.php/da/praehospital

[21] S. Thelen, M.-T. Schneiders, D. Schilberg, and S. Jeschke, "A multifunctional telemedicine system for pre-hospital emergency medical services," in Proc. 5th Int. Conf. eHealth, Telemed., Social Med., 2013, pp. 53-58.

[22] Philips Healthcare. (2014, Mar. 03). IntelliVue Information Center System Release L.0: Installation and Service Manual. [Online]. Available: http://incenter.medical.philips.com/doclib/getDoc.aspx?func $=11 \&$ objId $=$ 8673388\&objAction $=$ Open

[23] Philips Healthcare. (2014, Feb. 28). HeartStart Telemedicine System User Guide. [Online]. Available: http://incenter.medical.philips.com/ doclib/getDoc.aspx ? func $=11 \&$ objId $=9286003 \&$ objAction $=$ Open

[24] T. Brodziak, I. Forkel, P. Irla, P. Kornatowski, and P. Seidenberg. (2013, Apr. 21). Secure and Reliable Communication for Telemedical Applications in Emergency Medical Services. Presented at ICT meets Medicine and Health (ICTMH2013) Workshop. [Online]. Available: http://www.comnets.uni-bremen.de/cewit-tzi-workshop2013/PDF/papers/Brodziak.pdf

[25] deba81, mknambiar, and pravinpardeshi. (2011, Mar.). Wide Area Network Emulator. WANem Virtual Appliance. [Online]. Available: http://sourceforge.net/projects/wanem/

[26] F. Vacirca, F. Ricciato, and R. Pilz, "Large-scale RTT measurements from an operational UMTS/GPRS network," in Proc. 1st Int. Conf. Wireless Internet, 2005, pp. 190-197.

Author's photographs and biographies not available at the time of publication. 